УДК 621.643.001:536.2

\title{
ЧИСЛЕННЫЙ АНАЛИЗ ВЛИЯНИЯ НЕСТАЦИОНАРНОСТИ ПРОЦЕССОВ ТЕПЛОПЕРЕНОСА В ЗОНАХ РАЗМЕЩЕНИЯ ПОДЗЕМНЫХ ТЕПЛОПРОВОДОВ НА ИХ ТЕПЛОВЫЕ РЕЖИМЫ И ТЕПЛОВЫЕ ПОТЕРИ
}

\author{
Половников Вячеслав Юрьевич, \\ polov@tpu.ru \\ Национальный исследовательский Томский политехнический университет, \\ Россия, 634050, г. Томск, пр. Ленина, 30.
}

\begin{abstract}
Актуальность исследования обусловлена необходимостью разработки новых подходов к анализу тепловых режимов и тепловых потерь подземных трубопроводов и подтверждается основными положениями Энергетической стратегии России на период до 2030 г. Подземные трубопроводы широко используются при транспортировке жидкостей в различных областях, например, таких как водоснабжение и теплоснабжение, нефтепроводы и газопроводы, технологические трубопроводы промышленных предприятий. При проектировании систем такого рода необходимо учитывать нестационарность теплообмена между грунтом и подземным трубопроводом, что во многих случаях оказывает существенное влияние на экономичность транспортировки энергоносителей.

Цель: численный анализ нестационарных тепловых режимов и тепловых потерь подземных теплоопроводов и исследование температурных полей и закономерностей нестационарного теплопереноса в зонах размещения подземных бесканальных трубопроводов.

Объекты: типичные для систем транспортировки энергоносителей подземные двухтрубные бесканальные трубопроводы. Трубопроводы изолированы пенополиуретаном и защитным покровным гидроизоляционным слоем из полиэтилена. Температуры на внутренней поверхности труб равны среднегодовым температурам энергоносителей в подающих и обратных трубопроводах водяных тепловых сетей при их работе по температурному графику $95 / 70{ }^{\circ} \mathrm{C}$. Сезонное изменение температуры окружающей среды вычислялось по закону простого гармонического колебания. Исследования проводились для климатических условий г. Томск. Средний коэффициент теплоотдачи на поверхности раздела «грунт - окружающая среда» составлял 5 BT/( M $\left.^{2} \cdot \mathrm{K}\right)$.

Методы: численное решение задач теплопереноса методом конечных элементов с использованием аппроксимации Галеркина, неравномерной конечно-элементной сетки, количество элементов которой выбирается из условий сходимости решения, сгущение сетки проводится методом Делоне.

Результаты. Установлены масштабы тепловых потерь и закономерности нестационарного теплопереноса в зонах размещения подземных бесканальных трубопроводов. Показана необходимость учета нестационарности теплопереноса в зоне размещения подземных теплопроводов при прогностическом моделировании их тепловых режимов и тепловых потерь. Нестационарность теплопереноса в зоне размещения подземных тепловых сетей может оказать заметное влияние, например в тех случаях, когда в зоне теплового влияния подземных трубопроводов расположены смежные коммуникации или инженерные сооружения.
\end{abstract}

\section{Ключевые слова:}

Системы транспортировки тепловой энергии, подземные трубопроводы,

тепловые потери, математическое моделирование, нестационарный теплоперенос.

\section{Введение}

Современные системы централизованного теплоснабжения объединяют передовые технологии оперативного регулирования тепловых режимов и минимизации эксплуатационных затрат [1]. Основные преимущества современных сетей централизованного теплоснабжения заключаются в их высокой энергоэффективности [2] и низких выбросах парниковых газов [3]. Повсеместный рост потребления энергии, необходимость повышения энергобезопасности, высокая стоимость энергии и ряд экологических факторов стали причинам того, что в последние годы заметно выросло количество сетей централизованного теплоснабжения во всем мире [2].

Развитие систем централизованного теплоснабжения неразрывно связано с исследованиями тепловых режимов и тепловых потерь тепловых сетей. Исследования большой группы авторов [4-10] показывают, что высокая степень детализации анализа основных физических процессов даже для таких относительно простых систем, как теплопроводы (и процессов тепло- и массопереноса в них), может создать объективные предпосылки для повышения энергоэффективности работы технологических устройств и систем, предназначенных для производства, транспорта, распределения и использования теплоты. Учет комплекса взаимосвязанных факторов и процессов, оказывающих существенное влияние на уровень тепловых потерь [4-10], позволяет на стадии проектирования или реконструкции тепловых сетей минимизировать потери тепловой энергии и продолжительности переходных процессов, и, следовательно, заметно повысить энергоэффективность транспортировки теплоносителей.

Известно, что численные методы решения задач тепло- и массопереноса в конструкциях и зонах размещения тепловых сетей обеспечивают достаточно точное прогнозирование тепловых режимов и тепловых потерь рассматриваемых систем $[4,11]$. При этом можно использовать как коммерческие программные комплексы $[5,6,8]$, так и оригинальные программные коды, написанные на высокоуровневых языках программирования $[7,9,10]$. 
Стационарные модели теплового состояния тепловой сети широко используются при прогностическом моделировании [12-14] и оптимизации работы сетей теплоснабжения $[15,16]$. Однако такие модели [9, 12-16] не описывают переходные процессы, которые при определенных условиях $[10,17-20]$ могут оказывать существенное влияние на интенсификацию тепловых потерь рассматриваемых объектов.

Целью данной работы является численный анализ влияния нестационарности процессов теплопереноса в зонах размещения подземных теплопроводов на их тепловые режимы и тепловые потери.

\section{Постановка задачи}

Рассматривается типичная бесканальная прокладка тепловой сети - трубопроводы, изолированные пенополиуретаном и защитным покровным гидроизоляционным слоем из полиэтилена [9]. Cхематическое изображение области решения приведено на рис. 1 и соответствует области решения для задачи, описанной в [9]. Для рассматриваемой области (рис. 1) решается двумерная нестационарная задача теплопереноса в системе «подземный бесканальный теплопровод - окружающая среда» .

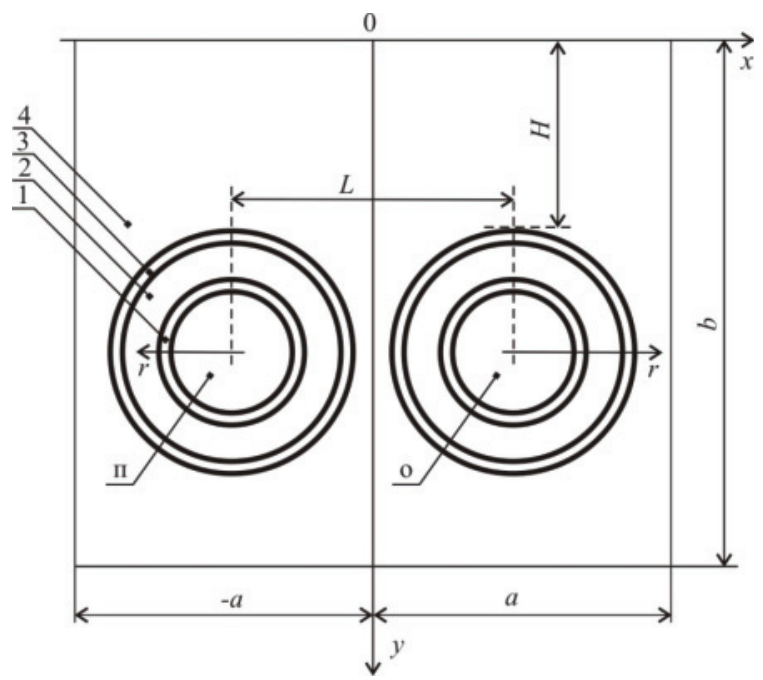

Рис. 1. Схема области решения: 1 - металлическая стенка трубы; 2 - теплоизоляционный слой; 3 - слой гидроизоляции; 4 - грунт; п, о - подающий и обратный теплопроводы; Н - расстояние от поверхности грунта до верхних точек слоев гидроизоляции; L - расстояние между осями трубопроводов; $a, b-$ размеры области решения

Fig. 1. Scheme of solution region: 1 is the pipe metal wall; 2 is the heat-insulating layer; 3 is the waterproofing layer; 4 is the soil; $n, 0$ are the supply and return heat pipes; $H$ is the distance from the soil surface to the upper points of the waterproofing layers; $L$ is the distance between the axes of the pipes; $a, b$ are the sizes of the solution region

\section{Математическая модель}

Математическая постановка задачи для рассматриваемой области решения (рис. 1) будет описываться нестационарными уравнениями теплопровод- ности в цилиндрической системе координат для стенок труб, теплоизоляционных и гидроизоляционных слоев подающего и обратного трубопроводов:

$$
\begin{gathered}
C_{i} \rho_{i} \frac{\partial T_{i, \mathrm{I}}}{\partial \tau}=\lambda_{i}\left(\frac{\partial^{2} T_{i, \mathrm{I}}}{\partial r^{2}}+\frac{1}{r} \frac{\partial T_{i, \mathrm{I}}}{\partial r}\right), i=1-3, \\
C_{i} \rho_{i} \frac{\partial T_{i, \mathrm{o}}}{\partial \tau}=\lambda_{i}\left(\frac{\partial^{2} T_{i, \mathrm{o}}}{\partial r^{2}}+\frac{1}{r} \frac{\partial T_{i, 0}}{\partial r}\right), i=1-3 ;
\end{gathered}
$$

в грунте нестационарным уравнением теплопроводности в декартовой системе координат:

$$
C_{4} \rho_{4} \frac{\partial T_{4}}{\partial \tau}=\lambda_{4}\left(\frac{\partial^{2} T_{4}}{\partial x^{2}}+\frac{\partial^{2} T_{4}}{\partial y^{2}}\right) .
$$

В начальный момент времени температура в рассматриваемой области решения являлась постоянной и известной величиной:

$$
T_{i}=T_{\mathrm{H}}=\text { const, } i=1-4, \tau=0 .
$$

При постановке задачи принималось, что во время отопительного периода на внутренних поверхностях труб подающего и обратного трубопроводов поддерживается постоянная температура, равная температуре теплоносителя в трубе:

$$
T_{1, \mathrm{\Pi}, \mathrm{o}}=T_{\mathrm{п}, \mathrm{o}}=\text { const, } 0<\tau \leq \tau_{\mathrm{oc}},
$$

а в межотопительный период на внутренней поверхности труб тепловой поток был равен нулю:

$$
\frac{\partial T_{1, \mathrm{n}, \mathrm{o}}}{\partial r}=0, \quad \tau_{\mathrm{oc}}<\tau \leq \tau_{0} .
$$

На границах между цилиндрическими слоями реализуются условия идеального теплового контакта:

$\lambda_{i} \frac{\partial T_{i, \mathrm{\Pi}, \mathrm{o}}}{\partial r}=\lambda_{j} \frac{\partial T_{j, \Pi, \mathrm{o}}}{\partial r}, T_{i, \mathrm{\Pi}, \mathrm{o}}=T_{j, \mathrm{\Pi \Pi}, \mathrm{o}}, \quad i, j=1-3 ; i \neq j ; \tau>0$.

Граничные условия для грунта в местах закладки подающего и обратного теплопроводов имеют следующий вид:

$$
\lambda_{3} \frac{\partial T_{3, \Pi, \mathrm{o}}}{\partial r}=\lambda_{4} \frac{\partial T_{4, \mathrm{\Pi}, \mathrm{o}}}{\partial r}, \quad T_{3, \mathrm{\Pi}, \mathrm{o}}=T_{4, \mathrm{\Pi}, \mathrm{o}} ;
$$

при условии, что $\tau>0$

$$
\begin{gathered}
\frac{L}{2}-R_{3} \leq x \leq \frac{L}{2}+R_{3} ; \quad H \leq y \leq H+2 R_{3} ; \\
R_{3}^{2}=\left(\frac{L}{2}+R_{3}-x\right)^{2}+(y-H)^{2} .
\end{gathered}
$$

На левой и правой границах рассматриваемой системы (рис. 1) градиенты температур в грунте равны нулю:

$$
\frac{\partial T_{4}}{\partial x}=0 \text {, при } \tau>0 .
$$

На поверхности раздела «грунт - окружающая среда» реализуются условия конвективного теплообмена:

$$
-\lambda_{4} \frac{\partial T_{4}}{\partial y}=\alpha\left(T_{4, \mathrm{~s}}-T_{5}(\tau)\right), \text { при } y=0, \tau>0 .
$$


На нижней поверхности градиент температуры равен нулю:

$$
\frac{\partial T_{4}}{\partial y}=0 \text {, при } y=b, \tau>0 \text {. }
$$

Обозначения: $T$ - температура, $K ; x, y, r$ - координаты, м; $\tau$ - время, с; $\tau_{\text {ос }}$ длительность отопительного периода, с; $\tau_{0}$ - время расчета, c; $\lambda$ - коэффициент теплопроводности, Вт ( $\left(\mathrm{r}^{\circ} \mathrm{K}\right) ; \mathrm{C}$ - теплоемкость, Дж $/(\kappa г \cdot К) ; \rho-$ плотность кг $/ \mathrm{M}^{3} ; \alpha-$ коэффициент теплоотдачи, $\mathrm{B} \mathrm{T} /\left(\mathrm{M}^{2} \cdot \mathrm{K}\right) ; R_{3}$ - внешняя поверхность теплопровода; $1-4$ - номера областей расчета (рис. 1); 5 - окружающая среда; п, о - подающий и обратный трубопроводы; н - начальный момент времени; в - верхняя граница.

\section{Метод решения и исходные данные}

Задача (1)-(11) по аналогии с [9] решена методом конечных элементов с использованием аппроксимации Галеркина [21]. Исследования проводились на неравномерной конечно-элементной сетке. Их количество выбиралось из условий сходимости решения; сгущение сетки проводилось методом Делоне [21].

Основное отличие задачи (1)-(11) от задачи, описанной в [9], заключается в учете начального распределения температур, периодической работы теплопроводов и зависимости температуры окружающей среды $T_{5}$ от времени. Сезонное изменение температуры окружающей среды вычислялось по закону простого гармонического колебания [10]:

$$
T_{5}(\tau)=A \sin \left(\frac{\pi \tau}{\tau_{0}}\right)
$$

где $A$ - амплитуда колебания температуры наружного воздуха, К [22, 23].

Предполагалось, что регулирование тепловой нагрузки в сети осуществляется количественным способом, а температуры внутренних поверхностей трубопроводов в отопительном периоде равны $T_{\mathrm{n}}=338 \mathrm{~K}$ и $T_{0}=323 \mathrm{~K}$ (температурный график $\left.95 / 70{ }^{\circ} \mathrm{C}[22]\right)$. Амплитуда колебания температуры наружного воздуха при исследовании нестационарных тепловых режимов подземных теплопроводов принималась как разница между наивысшей и самой низкой температурами за год [23], а при решении стационарной задачи температура окружающей среды принималась равной средней температуре воздуха за отопительный период 264,2 К [22]. Исследования проводились для климатических условий г. Томск (табл. 1). Условно предполагалось, что отопительный период начинается в сентябре и заканчивается в апреле (табл. 1). Начальное распределение температур в рассматриваемой области решения (рис. 1) принималось равным $T_{\text {म }}=282,2 \mathrm{~K}$ и соответствовало значению температуры окружающей среды в начале отопительного периода. Средний коэффициент теплоотдачи на поверхности раздела «грунт - окружающая среда» составлял $5 \mathrm{~B} \mathrm{~T} /\left(\mathrm{M}^{2} \cdot \mathrm{K}\right)$.
Таблица 1. Среднемесячная температура воздуха для г. Томск [23]

\begin{tabular}{|c|c|c|c|c|c|c|c|c|c|c|c|c|}
\hline $\begin{array}{l}\text { Месяц } \\
\text { Month }\end{array}$ & 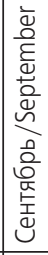 & 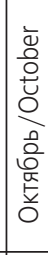 & 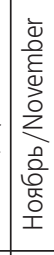 & 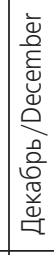 & 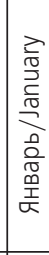 & 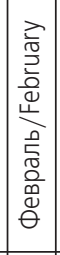 & 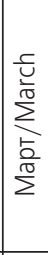 & 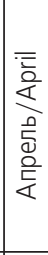 & 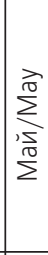 & 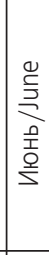 & 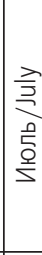 & 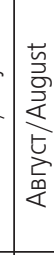 \\
\hline $\begin{array}{c}\text { Температура } \\
\text { воздуха, K } \\
\text { Air tempera- } \\
\text { ture, K }\end{array}$ & 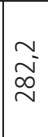 & $\begin{array}{l}\text { A } \\
\text { 齐 }\end{array}$ & 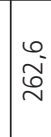 & 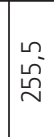 & 足 & $\begin{array}{l}m \\
\text { o } \\
\stackrel{\text { ڤn }}{n}\end{array}$ & o & I & 官 & $\begin{array}{l}m \\
\infty \\
\infty \\
\sim \\
N\end{array}$ & 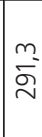 & $\begin{array}{l}\bar{\infty} \\
\infty \\
\sim\end{array}$ \\
\hline
\end{tabular}

Table 1. Average monthly air temperature for Tomsk [23]

При проведении численного моделирования в соответствии с [9] использовалась расчетная область размерами $b=-6$ м в глубину и $a= \pm 5$ м в стороны от оси симметрии (рис. 1). Исследования проводились для трубопроводов, конфигурация которых полностью соответствует описанной в [9]: диаметр условного прохода трубопроводов 600 мм, изготовлены из стали 10 (толщина стенки 8 мм), тепловая изоляция - пенополиуретан (толщина 40 мм), покровный слой - полиэтилен (2 мм). Расстояние от поверхности грунта до верхних точек слоев гидроизоляции принималось равным $H=2$ м, а между осями трубопроводов составляло $L=1,3$ м (рис. 1 ).

В табл. 2 приведены значения [24] теплопроводности $(\lambda)$, теплоемкости $(C)$ и плотности $(\rho)$ грунта, стенки трубы, тепловой и гидроизоляции, использовавшиеся при проведении численных исследований.

Таблица 2. Теплофизические характеристики [24]

\begin{tabular}{|c|c|c|c|c|}
\hline $\begin{array}{l}\text { Материал } \\
\text { Material }\end{array}$ & $\begin{array}{c}\text { Покровный } \\
\text { слой } \\
\text { Cover layer }\end{array}$ & $\begin{array}{l}\text { Тепловая изоляция } \\
\text { Thermal insulation }\end{array}$ & $\begin{array}{c}\text { Стенка } \\
\text { трубы } \\
\text { Pipe wall }\end{array}$ & $\begin{array}{c}\text { Грунт } \\
\text { Soil }\end{array}$ \\
\hline $\begin{array}{c}\lambda, B \mathrm{BT} /(\mathrm{M} \cdot \mathrm{K}) \\
\mathrm{W} /(\mathrm{m} \cdot \mathrm{K})\end{array}$ & 0,33 & 0,033 & 50,2 & 1,3 \\
\hline $\begin{array}{c}\text { C, Дж/(кг } \cdot \mathrm{K}) \\
\mathrm{J} /(\mathrm{kg} \cdot \mathrm{K})\end{array}$ & 2200 & 1470 & 462 & 959 \\
\hline $\begin{array}{c}\rho, \mathrm{K} \Gamma / \mathrm{M}^{3} \\
\mathrm{~kg} / \mathrm{m}^{3}\end{array}$ & 920 & 50 & 7700 & 1700 \\
\hline
\end{tabular}

Table 2. Thermophysical characteristics [24]

\section{Результаты численного моделирования}

Основные результаты численного анализа нестационарных тепловых режимов и тепловых потерь подземных бесканальных трубопроводов приведены на рис. 2-4.

Обоснованность и достоверность результатов исследований следует из проведенных проверок используемых методов на сходимость и устойчивость решений на множестве сеток, выполнения условий баланса энергии на границах области расчета, а также подтверждается хорошим качественным согласованием полученных результатов с известными данными других авторов, например 


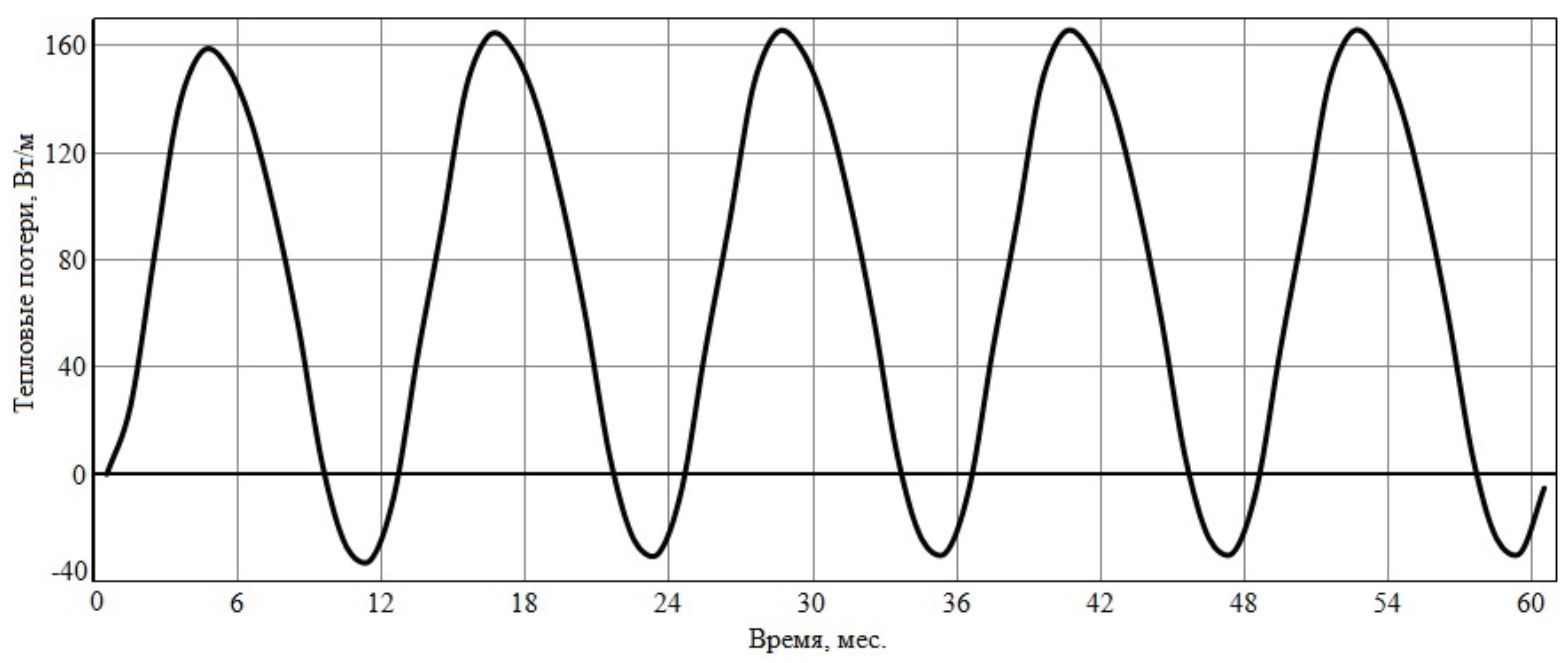

Рис. 2. Изменение тепловых потерь подземного бесканального теплопровода во времени

Fig. 2. Change in thermal losses of an underground non-channel heat pipe in time

$[4-8,10]$. Относительная погрешность расчетов во всех вариантах численного анализа не превышала $0,2 \%$, что является приемлемым при анализе тепловых режимов и тепловых потерь подземных трубопроводов.

На рис. 2 приведена характерная зависимость тепловых потерь от времени для подземного теплопровода рассматриваемой конфигурации (рис. 1). Исследования проводились для промежутка времени, равного пяти годам эксплуатации тепловой сети. Первое распределение температур в рассматриваемой области решения соответствовало условию (4), а в начале каждого последующего отопительного периода за начальное распределение температур принималось температурное поле в грунте и изоляционных конструкциях теплопроводов в конце предшествующего межотопительного сезона.

Результаты численного моделирования потерь тепловой энергии подземных бесканальных трубопроводов с учетом изменения температуры окружающей среды во времени, приведенные на рис. 2 , свидетельствуют об ожидаемом циклическом изменении тепловых потерь в зависимости от значений температур наружного воздуха (табл. 1) при фиксированных температурах теплоносителей в трубах (количественное регулирование тепловой нагрузки).

Максимальный диапазон изменения тепловых потерь рассматриваемой системы (рис. 1) составляет $197,71 \mathrm{Bт} /$ м от $165,54 \mathrm{Bт} /$ м в декабре и до минус 32,17 Вт/м в июле. При решении стационарной задачи теплопереноса для теплопровода с аналогичной конфигурацией и условиями эксплуатации тепловые потери составили 105,4 Вт/м, что хорошо согласуется с результатами исследования [9].

«Отрицательные» тепловые потери (теплоприток) наблюдаются в летний период и объясняются отсутствием теплового потока от трубопроводов в межотопительный период и нагревом грунта от внешней среды. По результатам численного моделирования было установлено, что средняя температура грунта в рассматриваемой области решения в конце межотопительного периода пятого года эксплуатации составляет: на поверхности $288 \mathrm{~K}$, на глубине $1 \mathrm{~m}-287,5 \mathrm{~K}$, а на глубине $6 \mathrm{~m}-285,5 \mathrm{~K}$. Такое распределение температур объясняется не только наличием теплового потока от внешней среды, но и в большей степени влиянием тепловой инерции грунта, в котором в отопительный период находились «разогретые» трубопроводы системы теплоснабжения.

Анализ результатов численного моделирования (рис. 2) позволяет сделать вывод о том, что начальные условия эксплуатации подземных бесканальных трубопроводов оказывают влияние на тепловые потери только в первый год работы тепловой сети и могут быть исключены из рассмотрения при прогностическом моделировании длительных периодов эксплуатации подземных трубопроводов.

На рис. 3-5, в качестве примеров, приведены типичные температурные поля в зоне прокладки бесканальных теплопроводов в первый, второй и третий годы эксплуатации для последнего дня работы в январе месяце.

Распределения температур в рассматриваемой области решения свидетельствуют о том, что изотермические линии (рис. 3-5) сгущаются непосредственно над подземными трубопроводами и более разрежены при удалении от них, что соответствует представлениям о процессах теплопроводности и качественно согласуется с результатами предыдущих исследований [9]. Рис. 3-5 наглядно демонстрируют существенное изменение температурного поля во времени в первые два года эксплуатации тепловой сети (рис. 3,4$)$. Начиная с третьего года работы подземных теплопроводов (рис. 5) изменение поля температур в рассматриваемой области решения в фиксированные моменты времени является незначительным. Необходимо от- 


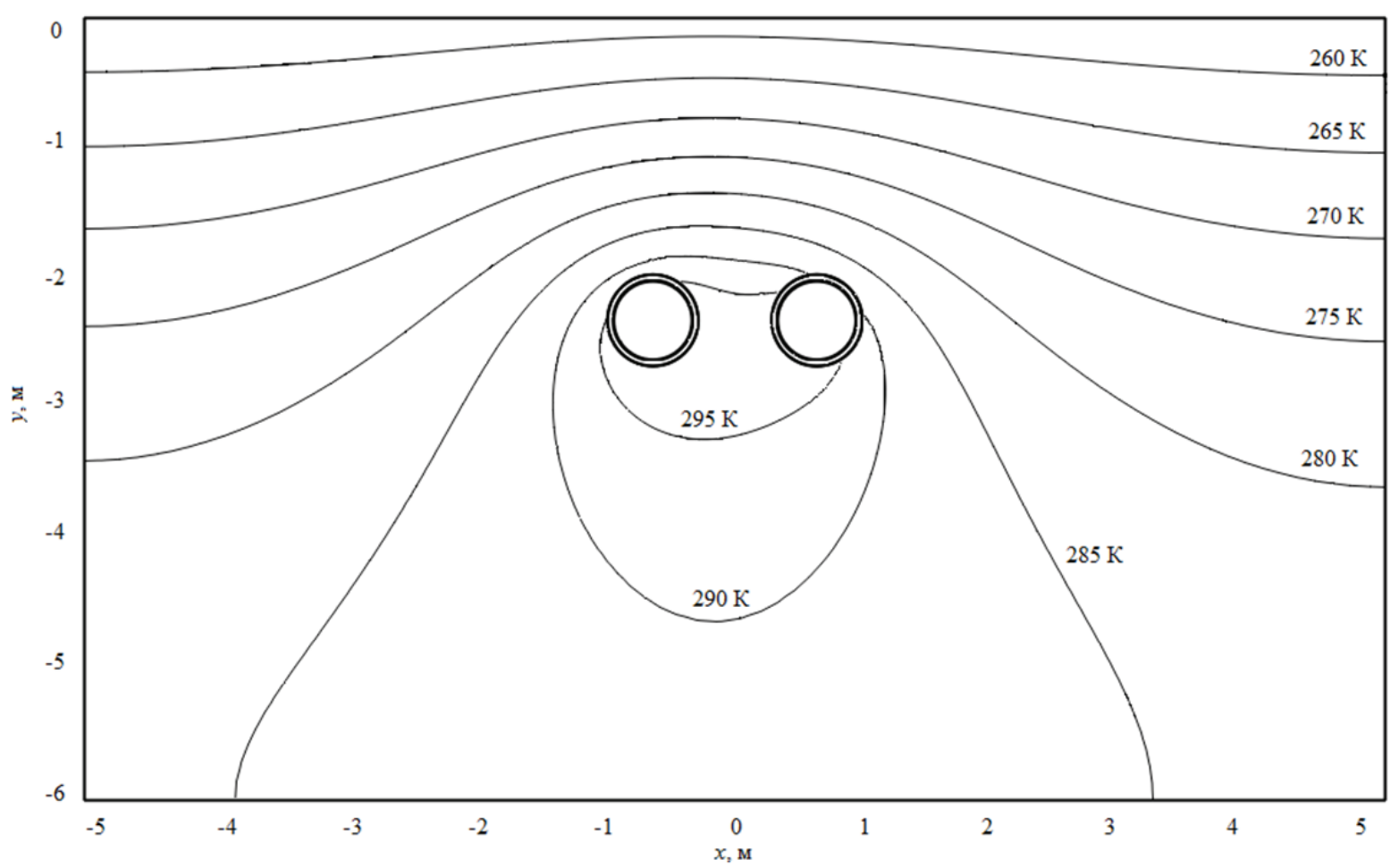

Рис. 3. Типичное температурное поле в зоне прокладки подземных трубопроводов (первый год эксплуатации)

Fig. 3. Typical temperature field in the zone of laying underground pipelines (the first year of operation)

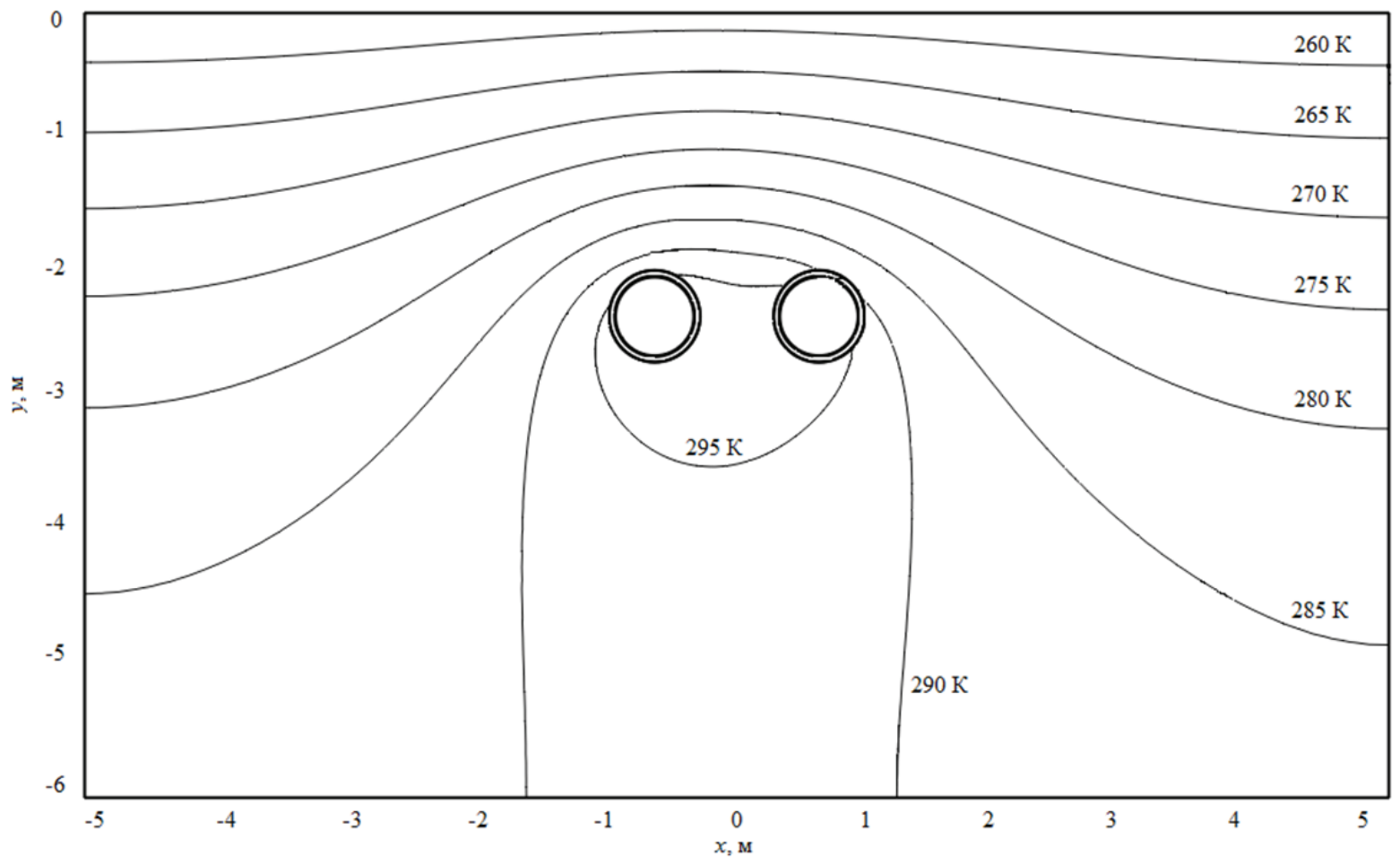

Рис. 4. Типичное температурное поле в зоне прокладки подземных трубопроводов (второй год эксплуатации)

Fig. 4. Typical temperature field in the zone of laying underground pipelines (the second year of operation) 


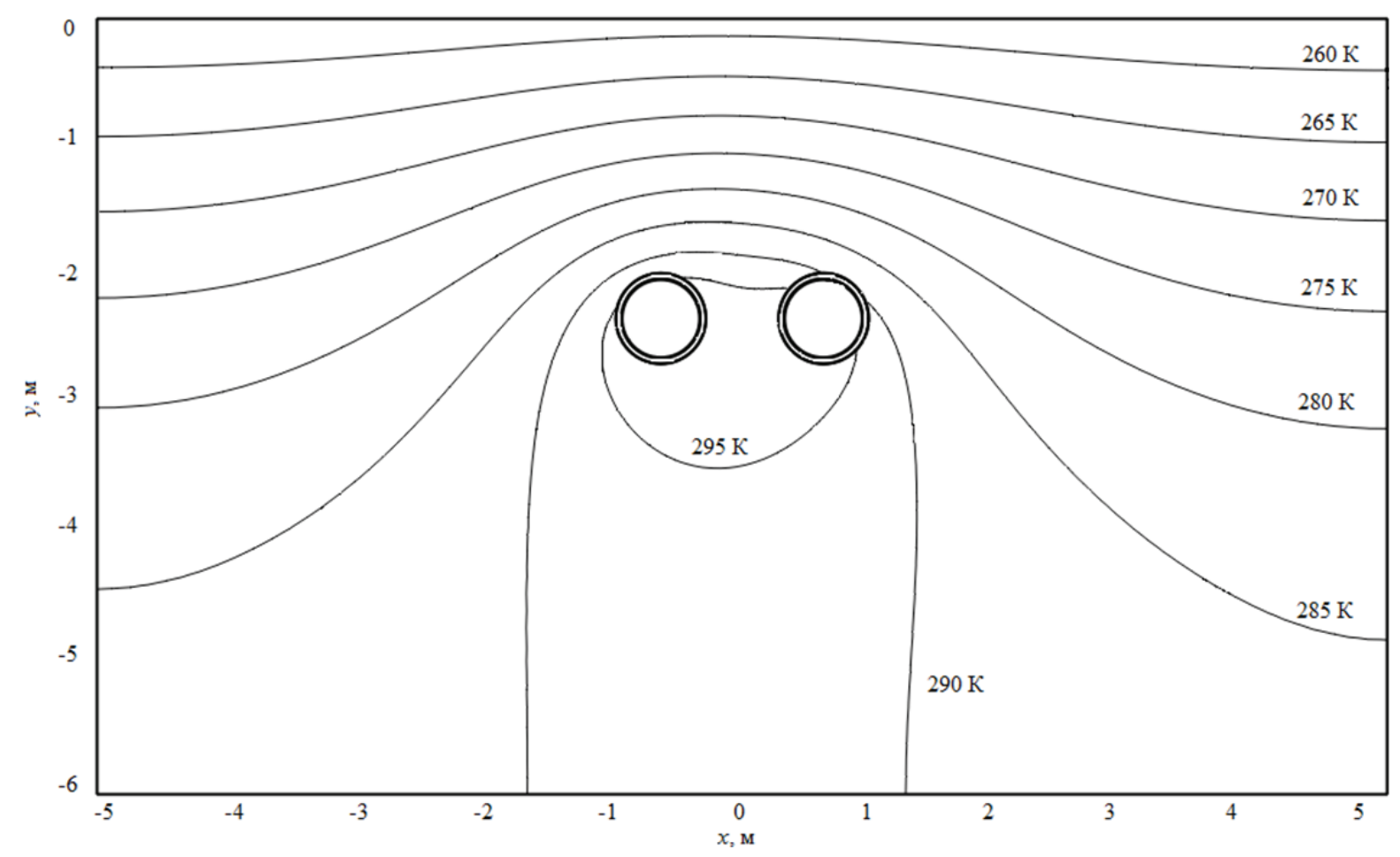

Рис. 5. Типичное температурное поле в зоне прокладки подземных трубопроводов (третий год эксплуатации)

Fig. 5. Typical temperature field in the zone of laying underground pipelines (the third year of operation)

метить, что в течение отопительного сезона из-за влияния переменной во времени температуры окружающей среды наблюдаются заметные изменения температурного поля грунта в месте прокладки подземных трубопроводов. Годовое смещение одноименных изотермических линий составляет от полуметра и более и увеличивается с уменьшением глубины.

Таким образом, тепловые режимы подземных трубопроводов являются существенно нестационарными и должны учитываться при прогностическом моделировании работы теплопроводов. Нестационарность теплопереноса в зоне размещения подземных тепловых сетей может оказать заметное влияние, например, в тех случаях, когда в зоне

\section{СПИСОК ЛИТЕРАТУРЫ}

1. Optimization modeling for smart operation of multi-source district heating with distributed variable-speed pumps / H. Wang, H. Wang, Zh. Haijian, T. Zhu // Energy. - 2017. - V. 138. P. $1247-1262$.

2. Duquette J., Rowe A., Wild P. Thermal performance of a steady state physical pipe model for simulating district heating grids with variable flow // Applied Energy. - 2016. - V. 178. P. 383-393.

3. Rezaie B., Rosen M. District heating and cooling: review of technology and potential enhancements // Applied Energy. 2012. - V. 93. - P. 2-10.

4. Thermal transient prediction of district heating pipeline: Optimal selection of the time and spatial steps for fast and accurate calculation / Y. Wanga, S. Youa, H., Zhanga X. Zhenga, W. Zhenga, теплового влияния подземных трубопроводов расположены смежные коммуникации или инженерные сооружения.

\section{Заключение}

Установлены масштабы тепловых потерь и закономерности нестационарного теплопереноса в зонах размещения подземных бесканальных трубопроводов.

Показана необходимость учета нестационарности теплопереноса в зоне размещения подземных теплопроводов при прогностическом моделировании их тепловых режимов и тепловых потерь.

Исследование выполнено при финансовой поддержке РФФИ в рамках научного проекта № 16-08-00229-а.

Q. Miaoa, G. Luc // Applied Energy. - 2017. - V. 206. P. $900-910$.

5. Soil thermal conductivity prediction for district heating pre-insulated pipeline in operation / M. Perpar, Z. Rek, S. Bajric, I. Zun // Energy. - 2012. - V. 44. - P. 197-210.

6. Three-dimensional numerical model of heat losses from district heating network pre-insulated pipes buried in the ground / J. Danielewicz, B. Sniechowska, M.A. Sayegh, N. Fidorow, H. Jouhara // Energy. - 2016. - V. 108. - P. 172-184.

7. Ertürk M. Optimum insulation thicknesses of pipes with respect to different insulation materials, fuels and climate zones in Turkey // Energy. - 2016. - V. 113. - P. 991-1003.

8. Gabrielaitiene I., Bøhm B., Sunden B. Evaluation of Approaches for Modeling Temperature Wave Propagation in District Heating Pipelines // Heat Transfer Engineering. - 2008. - V. 29. № 1. - P. $45-56$. 
9. Половников В.Ю. Тепловые режимы и тепловые потери подземных трубопроводов с учетом реальных условий теплообмена на внешнем контуре взаимодействия // Известия Томского политехнического университета. Инжиниринг георесурсов. 2018. - T. 329. - № 1. - С. 124-131.

10. Акимов М.П., Мордовской С.Д., Старостин Н.П. Определение толщины теплоизоляции и заглубления подземного трубопровода теплоснабжения в многолетнемерзлых грунтах // Инженерно-строительный журнал. - 2014. - № 2 (46). - С. 14-23.

11. Benonysson A., Bøhm B., Ravn H.F. Operational optimization in a district heating system // Energy Conversion and Management. 1995. - V. 36. - № 5. - P. 297-314.

12. Liu X., Wu J., Jenkins N., Bagdanavicius A. Combined analysis of electricity and heat networks // Applied Energy. - 2016. V. 162. - P. 1238-1250.

13. Pan Z., Guo Q., Sun H. Interactions of district electricity and heating systems considering time-scale characteristics based on quasi-steady multi-energy flow // Applied Energy. - 2016. V. 167. - P. 230-243.

14. Dalla R.A., Li H., Svendsen S. Method for optimal design of pipes for low energy district heating, with focus on heat losses / Energy. - 2011. - V. 36. - № 5. - P. 2407-2418.

15. Sartor K., Quoilin S., Dewallef P. Simulation and optimization of a CHP biomass plant and district heating network // Applied Energy. - 2014. - V. 130. - P. 474-483.
16. Fang T., Lahdelma R. Genetic optimization of multi-plant heat production in district heating networks // Applied Energy. 2015. - V. 159. - P. 610-619.

17. Bøhm B. Experimental determination of heat losses from buried district heating pipes in normal operation // Heat Transfer Engineering. - 2001. - V. 22. - № 3. - P. 41-51.

18. Janssen H., Carmeliet J., Hens H. The influence of soil moisture transfer on building heat loss via the ground // Building and Environment. - 2004. - V. 39. - № 7. - P. 825-836.

19. Bøhm B. On transient heat losses from buried district heating pipes // International Journal of Energy Research. - 2000. V. 24. - № 15. - P. 1311-1334.

20. Verda V., Colella F. Primary energy savings through thermal storage in district heating networks // Energy. - 2011. - V. 36. № 7. - P. 4278-4286.

21. Garcia A.L. Numerical methods for physics. - San Jose CA: CreateSpace Independent Publishing Platform, 2015. - 432 c.

22. Соколов Е.Я. Теплофикация и тепловые сети. - М.: Изд-во МЭИ, 2001. -472 c.

23. СНиП 2.01.01-82. Строительная климатология и геофизика. М.: Стройиздат, 1983. - 57 с.

24. Гува А.Я. Краткий теплофизический справочник. - Новосибирск: Сибвузиздат, 2002. - 300 с.

Поступила 7.03.2018 г.

\section{Информация об авторах}

Половников В.Ю., кандидат технических наук, доцент Инженерной школы энергетики Национального исследовательского Томского политехнического университета. 
UDK 621.643.001:536.2

\title{
NUMERICAL ANALYSIS OF NONSTATIONARY HEAT TRANSFER INFLUENCE IN THE ZONES OF UNDERGROUND THERMAL PIPELINES ON THEIR THERMAL REGIMES AND HEAT LOSSES
}

\author{
Viacheslav Yu. Polovnikov, \\ polov@tpu.ru \\ National Research Tomsk Polytechnic University, \\ 30, Lenin Avenue, Tomsk, 634050, Russia.
}

The relevance of the research is caused by the need to develop new approaches to the study of thermal regimes and thermal losses of underground pipelines and it is confirmed by the main provisions of the Energy Strategy of Russia for the period up to 2030. Underground pipelines are widely used for liquid transportation in various areas, for example, water supply and heat supply, oil pipelines and gas pipelines, technological pipelines of industrial enterprises. When designing an underground piping system, it is necessary to take into account nonstationary heat exchange between the ground and the underground pipeline. In many cases this impacts significantly the economics of transportation of energy carriers.

The main aim of the research is a numerical analysis of nonstationary thermal conditions and heat losses of underground channel-free pipelines and investigation of temperature fields and patterns of nonstationary heat transfer in the areas of placement of underground non-channel pipelines.

Objects of the research are the typical for energy transportation system underground two-pipe ductless pipelines. Pipelines are insulated with polyurethane foam and protective covering waterproofing layer made of polyethylene. Temperature of energy carriers is equal to the average annual temperature of the energy carriers in the supply and return pipelines of the water heating networks during their operation according to the temperature schedule $95 / 70{ }^{\circ} \mathrm{C}$. The seasonal change in the ambient temperature was calculated according to the law of a simple harmonic oscillation. The investigations were carried out for the climatic conditions of Tomsk. The average heat transfer coefficient at the ground-to-environment interface was $5 \mathrm{~W} /\left(\mathrm{m}^{2} \cdot \mathrm{K}\right)$.

Methods: numerical solution of heat transfer problems by the finite element method using the Galerkin approximation, non-uniform finite element mesh, the number of elements of mesh is chosen from the conditions of convergence of the solution; the grid is thickened by the Delaunay method.

Results. Scales of heat losses and regularities of nonstationary heat transfer in the zones of underground non-channel pipelines were established. The paper demonstrates the need to take into account the nonstationarity of heat transfer in the zone of underground heat pipes with predictive modeling of their thermal regimes and heat losses. Nonstationary heat transfer in the zone of underground heating networks can have a significant effect, for example, in cases where adjacent communications or engineering facilities are located in the zone of thermal influence of underground pipelines.

\section{Key words:}

Thermal energy transportation system, underground pipelines, heat losses, mathematical modeling, nonstationary heat transfer.

The research was financially supported by the RFBR within the scientific project no. 16-08-00229-a.

\section{REFERENCES}

1. Wang H., Wang H., Haijian Zh., Zhu T. Optimization modeling for smart operation of multi-source district heating with distributed variable-speed pumps. Energy, 2017, vol. 138, pp. 1247-1262.

2. Duquette J., Rowe A., Wild P. Thermal performance of a steady state physical pipe model for simulating district heating grids with variable flow. Applied Energy, 2016, vol. 178, pp. 383-393.

3. Rezaie B., Rosen M. District heating and cooling: review of technology and potential enhancements. Applied Energy, 2012, vol. 93, pp. 2-10.

4. Wanga Y., Youa S., Zhanga H., Zhenga X., Zhenga W., Miaoa Q., Luc G. Thermal transient prediction of district heating pipeline: Optimal selection of the time and spatial steps for fast and accurate calculation. Applied Energy, 2017, vol. 206, pp. 900-910.

5. Perpar M., Rek Z., Bajric S., Zun I. Soil thermal conductivity prediction for district heating pre-insulated pipeline in operation. Energy, 2012, vol. 44, pp. 197-210.

6. Danielewicz J., Sniechowska B., Sayegh M.A., Fidorow N., Jouhara H. Three-dimensional numerical model of heat losses from district heating network pre-insulated pipes buried in the ground. Energy, 2016, vol. 108, pp. 172-184.

7. Ertürk M. Optimum insulation thicknesses of pipes with respect to different insulation materials, fuels and climate zones in Turkey. Energy, 2016, vol. 113, pp. 991-1003.
8. Gabrielaitiene I., Bøhm B., Sunden B. Evaluation of Approaches for Modeling Temperature Wave Propagation in District Heating Pipelines. Heat Transfer Engineering, 2008, vol. 29, no. 1, pp. $45-56$.

9. Polovnikov V.Yu. Thermal regimes and thermal losses of underground pipelines in real heat exchange on the outer interaction boundary. Bulletin of the Tomsk Polytechnic University. Geo Assets Engineering, 2018, vol. 329, no. 1, pp. 124-131. In Rus.

10. Akimov M.P., Mordovskoy S.D., Starostin N.P. Calculating thermal insulation thickness and embedment depth of underground heat supply pipeline for permafrost soils. Magazine of Civil Engineering, 2014, vol. 46, no. 2, pp. 14-23. In Rus.

11. Benonysson A., Bøhm B., Ravn H.F. Operational optimization in a district heating system. Energy Conversion and Management, 1995, vol. 36, no. 5, pp. 297-314.

12. Liu X., Wu J., Jenkins N., Bagdanavicius A. Combined analysis of electricity and heat networks. Applied Energy, 2016, vol. 162, pp. 1238-1250.

13. Pan Z., Guo Q., Sun H. Interactions of district electricity and heating systems considering time-scale characteristics based on quasi-steady multi-energy flow. Applied Energy, 2016, vol. 167, pp. 230-243.

14. Dalla R.A., Li H., Svendsen S. Method for optimal design of pipes for low energy district heating, with focus on heat losses. Energy, 2011, vol. 36, no. 5, pp. 2407-2418. 
15. Sartor K, Quoilin S, Dewallef P. Simulation and optimization of a CHP biomass plant and district heating network. Applied Energy, 2014, vol. 130, pp. 474-483.

16. Fang T., Lahdelma R. Genetic optimization of multi-plant heat production in district heating networks. Applied Energy, 2015, vol. 159 , pp. 610-619.

17. Bøhm B. Experimental determination of heat losses from buried district heating pipes in normal operation. Heat Transfer Engineering, 2001, vol. 22, no. 3, pp. 41-51.

18. Janssen H., Carmeliet J., Hens H. The influence of soil moisture transfer on building heat loss via the ground. Building and Environment, 2004, vol. 39, no. 7, pp. 825-836.

19. Bøhm B. On transient heat losses from buried district heating pipes. International Journal of Energy Research, 2000, vol. 24, no. 15, pp. 1311-1334.
20. Verda V., Colella F. Primary energy savings through thermal storage in district heating networks. Energy, 2011, vol. 36, no. 7, pp. 4278-4286.

21. Garcia A.L. Numerical methods for physics. San Jose CA, CreateSpace Independent Publishing Platform, 2015. 432 p.

22. Sokolov E.Ya. Teplofikatsiya i teplovyie seti [District heating and heat networks]. Moscow, MEI Publ., 2001. 472 p.

23. SNiP 2.01.01-82. Stroitelnaya klimatologiya i geofizika [Building regulations 2.01.01-82. Building Climatology and Geophysics]. Moscow, Stroyizdat Publ., 1983. 57 p.

24. Guva A.Ya. Kratkiy teplofizicheskiy spravochnik [Brief thermophysical reference book]. Novosibirsk, Sibvuzizdat Publ., 2002. $300 \mathrm{p}$.

Received: 7 March 2018.

\section{Information about the authors}

Viacheslav Yu. Polovnikov, Cand. Sc., associate professor, National Research Tomsk Polytechnic University. 